\title{
Distribution of XTH, expansin, and secondary-wall-related CesA in floral and fruit abscission zones during fruit development in tomato (Solanum lycopersicum)
}

\author{
Mutsumi Tsuchiya $^{\dagger}$, Shinobu Satoh and Hiroaki Iwai ${ }^{\star \dagger}$ \\ Faculty of Life and Environmental Sciences, University of Tsukuba, Tsukuba, Japan
}

OPEN ACCESS

Edited by:

Timothy J. Tranbarger, Institut de recherche pour

le développement (IRD), France

Reviewed by:

Masatoshi Yamaguchi, Saitama University, Japan

Toshihisa Kotake,

Saitama University, Japan

Akira Tateishi,

Nihon University, Japan

*Correspondence:

Hiroaki Iwai,

Faculty of Life and Environmental Sciences, University of Tsukuba,

Tsukuba, Ibaraki 305-8572, Japan iwai.hiroaki.gb.@u.tsukuba.ac.jp

${ }^{\dagger}$ These authors have contributed equally to this work.

Specialty section:

This article was submitted to Crop Science and Horticulture, a section of the journal Frontiers in Plant Science

Received: 13 March 2015 Accepted: 24 April 2015 Published: 15 May 2015

Citation:

Tsuchiya M, Satoh S and Iwai H (2015) Distribution of XTH, expansin, and secondary-wall-related CesA in floral and fruit abscission zones during fruit development in tomato (Solanum

lycopersicum).

Front. Plant Sci. 6:323.

doi: $10.3389 /$ fpls.2015.00323
After fruit development is triggered by pollination, the abscission zone (AZ) in the fruit pedicel strengthens its adhesion to keep the fruit attached. We previously reported that xyloglucan and arabinan accumulation in the AZ accompanies the shedding of unpollinated flowers. After the fruit has developed and is fully ripened, shedding occurs easily in the AZ due to lignin accumulation. Regulation of cell wall metabolism may play an important role in these processes, but it is not well understood. In the present report, we used immunohistochemistry to visualize changes in the distributions of xyloglucan and arabinan metabolism-related enzymes in the AZs of pollinated and unpollinated flowers, and in ripened fruits. During floral abscission, we observed a gradual increase in polyclonal antibody labeling of expansin in the AZ. The intensities of LM6 and LM15 labeling of arabinan and xyloglucan, respectively, also increased. However, during floral abscission, we observed a large 1 day post anthesis (DPA) peak in the polyclonal antibody labeling of $\mathrm{XTH}$ in the $\mathrm{AZ}$, which then decreased. These results suggest that expansin and XTH play important, but different roles in the floral abscission process. During fruit abscission, unlike during floral abscission, no AZ-specific expansin and XTH were observed. Although lignification was seen in the $A Z$ of over-ripe fruit pedicels, secondary cell wall-specific cellulose synthase signals were not observed. This suggests that cellulose metabolism-related enzymes do not play important roles in the AZ prior to fruit abscission.

Keywords: abscission, XTH, expansin, fruit, flower, tomato

\section{Introduction}

Abscission is a process by which plants shed unnecessary organs such as leaves, flowers, and fruits to save metabolic energy, to protect themselves from biotic and abiotic stresses by releasing organs attacked by pathogens, and to allow seed dispersal by releasing fruits. Organ abscission occurs at a specialized region in the leaf stem and pedicel, known as the AZ. The AZ consists of several layers of microscopic cells that are distinct from surrounding cells, and which form well before organ separation (Roberts et al., 2000, 2002). Earlier studies have identified several MADS-box genes that are related to the formation of AZs, including JOINTLESS, a gene essential for AZ formation in tomato pedicels (Mao et al., 2000). Furthermore, one of the key enzymes in cell wall degradation, 
polygalacturonase, was first found in the tomato fruit pedicel AZ. Thus, the tomato plant has become an important resource in organ abscission research (Taylor et al., 1991; Kalaitzis et al., 1995, 1997). We previously reported that during floral abscission, a large increase was observed in LM15-labeling of xyloglucan specifically at the $\mathrm{AZ}$ in the abscised pedicel. During fruit abscission, unlike in floral abscission, we did not observe any AZ-specific cell wall polysaccharide deposition. However, high autofluorescence was seen in the AZ of overripe fruit pedicels, suggesting secondary cell wall synthesis and lignification of the AZ prior to fruit abscission (Iwai et al., 2013). In the previous report, we did not analyze enzymatic activities of cell wall modeling enzymes, such as xyloglucan endotransglucosylase/hydrolase (XTH), expansin, and certain cell wall synthesis-related enzymes (secondary cell wall enzymes: CesA4, 7, and 8). Biochemical experiments could not be performed due to the limited number of $\mathrm{AZ}$ samples, making enzymatic analyses quite difficult. Therefore, in this report, we visualized XTH, expansin, and cellulose synthase using immunohistochemistry.

Xyloglucan is one of the major hemicelluloses of primary cell walls, in dicot plants, and may account for up to $10-20 \%$ of cell wall components (Fry, 1989; Hayashi, 1989). They tether cellulose microfibrils by cross-linking them through non-covalent linkages, thus providing strength to the walls during growth. Modification in the length of xyloglucans during cell expansion is primarily mediated by the enzyme XTH through endotransglycosylation, thus enabling the cell wall to expand without weakening (Smith and Fry, 1991; Fry et al., 1992; Nishitani and Tominaga, 1992). XTHs belong to a multigene family (Xu et al., 1996; Campbell and Braam, 1999; Rose et al., 2002; Yokoyama et al., 2004), which plays important roles in several different processes during cell wall modification. These include root hair initiation (Vissenberg et al., 2000, 2001), hypocotyl elongation (Potter and Fry, 1994; Catalá et al., 1997, 2001), hydrolysis of seed storage carbohydrates (de Silva et al., 1993), leaf growth and expansion (Schunmann et al., 1997), aerenchyma formation (Saab and Sachs, 1996), fruit softening (Schroder et al., 1998; Ishimaru and Kobayashi, 2002; Saladié et al., 2006), tension wood formation (Nishikubo et al., 2007, 2011), and petal abscission (Singh et al., 2011). Expansins were the first proteins characterized that directly induce the extension of the plant cell wall (McQueen-Mason et al., 1992), and are believed to be important regulators of wall extension during plant cell growth (reviewed in Lee et al., 2001; Cosgrove et al., 2002; Li et al., 2003). Expansins appear to operate by disrupting hydrogen bonds between cellulose microfibrils and xyloglucans that tether them to one another in plant cell walls (McQueen-Mason and Cosgrove, 1994, 1995; Whitney et al., 2000). In addition to this role during cell growth, expansins play an important role in fruit softening (Rose et al., 1997; Brummell et al., 1999; Anjanasree and Bansal, 2003). Similarly, expansins are expressed at the point of radicle emergence in germinating tomato seeds (Chen and Bradford, 2000) and in the micropylar endosperm of Datura ferox seeds in response to red light (Mella et al., 2004), suggesting that they may play a general role in promoting cell wall dissolution. Abscission and fruit softening both involve cell wall breakdown, and many of the same types of enzymes are involved in the two processes (Rose and Bennett, 1999; Rose et al., 2003). Although there is some circumstantial evidence of an association between XTH and expansins and abscission (Cho and Cosgrove, 2000), no reports have been published showing a correlation between the activity of these proteins and organ shedding, especially during floral and fruit abscission.

In the present study, we present the first report that abscission is associated with elevated XTH and expansin, suggesting that these proteins contribute to the process of organ shedding. We also discuss the abscission systems that occur during floral and fruit abscission in tomato plants.

\section{Materials and Methods}

\section{Plant Material and Growth Conditions}

Tomato (Solanum lycopersicum cv Micro Tom) plants were grown inside a cultivation chamber (TOMY CL-301) under a $16 \mathrm{~h}$ light and $8 \mathrm{~h}$ dark regime, at temperatures of 26 and $22^{\circ} \mathrm{C}$, respectively, and a light intensity of approximately $100 \mu \mathrm{mol} \mathrm{m}^{-2} \mathrm{~s}^{-1}$.

\section{Pollination}

Tomato flowers were pollinated by hand. 1 day prior to flowering, the closed buds were opened using a pair of tweezers and the anthers were extracted, leaving only the pistil inside. The opened buds were pollinated the next day by rubbing a dehisced anther onto the stigma. Glassine paper bags were placed over the treated flowers at the time the anthers were extracted to avoid unwanted pollination and to protect against physical stress.

\section{Technovit Resin Sections}

Samples were fixed in $2.5 \%$ paraformaldehyde in $0.025 \mathrm{mM}$ phosphate-buffered saline (PBS) and evacuated using a vacuum pump for $12 \mathrm{~h}$. Fixed samples were dehydrated through the following series of EtOH concentrations: 30, 50, 70, 80, and $90 \%$ for $20 \mathrm{~min}$ each, and then 95 and 100\% twice for $30 \mathrm{~min}$. EtOH in dehydrated samples was exchanged for Technovit 7100 resin (Heraeus Kulzer, Wehrheim, Germany) through the following series of Technovit 7100:EtOH: 1:4, 2:3, 3:2, and 4:1 each for $30 \mathrm{~min}$, and then $100 \%$ Technovit for $30 \mathrm{~min}$ and $12 \mathrm{~h}$. Samples were then solidified in Technovit 7100 resin following the manufacturer's protocol. Embedded samples were cut into $5 \mu \mathrm{m}$ sections using a microtome and a glass knife.

\section{Paraffin Sections}

Samples were fixed in $4 \%$ paraformaldehyde at $4^{\circ} \mathrm{C}$ overnight for paraffin embedding. The fixed samples were dehydrated in a graded series of ethanol (70 and 85\%) followed by a 1-butanol/ethanol series (80\% ethanol/1-butanol 13:7, $90 \%$ ethanol/1-butanol 9:11, 100\% ethanol/1-butanol 1:3, and 100\% 1-butanol). 1-butanol was replaced gradually with paraffin (Paraplast Plus; McCormick Scientific, St. Louis, MO, USA) at $60^{\circ} \mathrm{C}$ over two nights inside an open jar to evaporate traces of $n$-butanol, and was then embedded in paraffin. Sections $12 \mu \mathrm{m}$ thick were cut using a rotary microtome (Leica RM2145), and the ribbons were placed and stretched out on albumin-glycerin-treated glass slides with distilled water (DW). The slides were dried at $45^{\circ} \mathrm{C}$ on a warming plate for 2 days, and stored at room temperature. For 
use, the slides were deparaffinized in xylene for $10 \mathrm{~min}$ (twice), and then hydrated in a graded series of ethanol (100, 90, 80, 70, and $0 \%$ in DW).

\section{Lignin Staining}

The sections were soaked in DW prior to staining. Phloroglucinol staining of lignin was performed according to Tadeo and PrimoMillo (1990). A saturated solution of phloroglucinol (SigmaAldrich) was prepared in $20 \% \mathrm{HCl}$ and applied to sections.

\section{Immunohistochemical Analysis}

Monoclonal rat IgG antibodies of LM6 and LM15 were purchased from PlantProbes (Leeds, $\mathrm{UK}^{1}$ ). Anti XTH, CesA4, CesA7, and CesA8 rabbit antibodies were obtained from Agrisera (Vännäs, Sweden ${ }^{2345}$ ). Anti expansin antibody was obtained from Biocompare (South San Francisco, San Mateo, CA, USA ${ }^{6}$ ). A TSA kit with HRP-conjugated secondary antibody and Alexa Fluor 488 tyramide were purchased from Invitrogen (Carlsbad, CA, USA; cat. \#T20912, \#20922, respectively). We used the set of polyclonal antibodies to perform immunohistochemistry according to the manufacturer's instructions. Briefly, the sections were incubated in PBS prior to labeling, and $100 \mu \mathrm{L}$ of the following reagents were added to the sections in consecutive order: quenching buffer (to quench endogenous peroxidase activity), 1\% blocking reagent, and primary antibody diluted in 1\% blocking reagent (1:30). After each addition, the sections were incubated at room temperature for $1 \mathrm{~h}$. The sections were then washed three times with PBS, incubated in $100 \mu \mathrm{L}$ horseradish peroxidase (HRP)-conjugated second antibody diluted in $1 \%$ blocking reagent $(1: 100)$ for $1 \mathrm{~h}$, again washed three times with PBS, incubated in $100 \mu \mathrm{L}$ Alexa Fluor 488 tyramide (excitation $495 \mathrm{~nm}$, emission $519 \mathrm{~nm}$ ) working solution (tyramide stock solution diluted in amplification buffer/0.0015\% $\mathrm{H}_{2} \mathrm{O}_{2} ; 1: 100$ ) for $10 \mathrm{~min}$ at room temperature, and finally washed three times with PBS and twice with DW. Immunofluorescence was visualized using a Leica DMRB fluorescence microscope, and all of the micrographs were captured with a DFC500 Leica digital camera using the IM50 Leica software (exposure time, $1.0 \mathrm{~s}$ ).

\section{Results}

\section{Immunolocalization of Xyloglucan and Arabinan Epitopes During -1 DPA Flower Pedicels to Yellowing Pedicels}

To investigate the distribution of cell-wall pectic arabinan and xyloglucan, we used their specific monoclonal antibodies to stain $-1 \mathrm{DPA}, 1 \mathrm{DPA}, 2 \mathrm{DPA}, 3 \mathrm{DPA}$ pedicels and yellowing pedicels

\footnotetext{
${ }^{1}$ www.plantprobes.net

${ }^{2}$ www.agrisera.com/en/index.html

${ }^{3} \mathrm{http}: / /$ www.agrisera.com/en/artiklar/cesa4-cellulose-synthase-a-catalyticsubunit-4-udp-forming.html

${ }^{4} \mathrm{http} / /$ www.agrisera.com/en/artiklar/cesa7-cellulose-synthase-a-catalyticsubunit-7-udp-forming.html

${ }^{5} \mathrm{http} / / /$ www.agrisera.com/en/artiklar/cesa8-cellulose-synthase-a-catalyticsubunit-8-udp-forming.html

${ }^{6} \mathrm{http} / / /$ www.biocompare.com/9776-Antibodies/2820012-anti-EXPB-2antibody
}

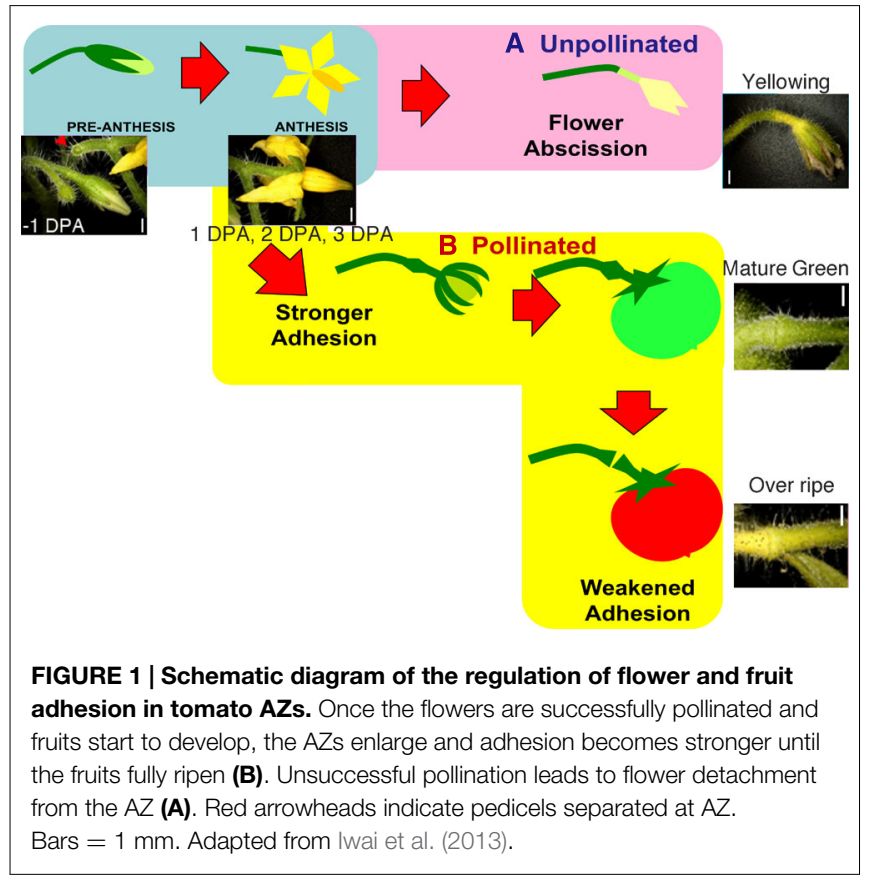

(Figure 1). Pedicels yellowed just before abscission. Immunodot assays and competitive inhibition ELISA performed by Willats et al. (1998) and Orfila and Knox (2000) indicated that the LM6 antibody is specific to $(1 \longrightarrow 5)$ - $\alpha$-L-linked arabinosyl residues with extended polymer, and reacts with rhamnogalacturonan (RG)-I but not RG-II. ELISA and glycan microarray performed by Marcus et al. (2008) indicated that LM15 binds specifically to xyloglucan, and competitive inhibition ELISAs determined that the structural feature for LM15 binding is the XXXG structural motif. Therefore, we used these probes to explore the occurrence of specific cell wall polysaccharides and differences in their distribution during early fruit development.

In the pollinated flower pedicel, the intensity of LM15 xyloglucan epitopes was low in -1 DPA to 3 DPA. The intensity of LM6 arabinan and LM15 xyloglucan epitopes was low throughout the tissue (Figure 2). Some weak signals were detected in vascular bundles by LM15; however, the labeling was not specific to the AZ.

In unpollinated flower pedicels, relative to $-1 \mathrm{DPA}$ pedicels, the intensity of LM6 pectic arabinan labeling increased in $3 \mathrm{DPA}$, and the highest signal was detected in the yellowing stage (Figure 3). The LM6 labeling was most intense at the AZ (Figure 3). Remarkably, LM15 labeling for xyloglucan epitopes increased dramatically, preferentially at the AZ (Figure 3). These results indicate that pectic arabinan and xyloglucan increase prior to abscission. As the time of abscission approached, accumulation of xyloglucan and arabinan increased (Figure 3).

\section{Immunolocalization of Expansin and XTH Epitopes During - 1 DPA Flower Pedicels to Yellowing Pedicels}

To investigate the potential role of XTH and expansin during the time course of floral abscission, we visualized changes in the distributions of XTH and expansin epitopes in -1 DPA, 


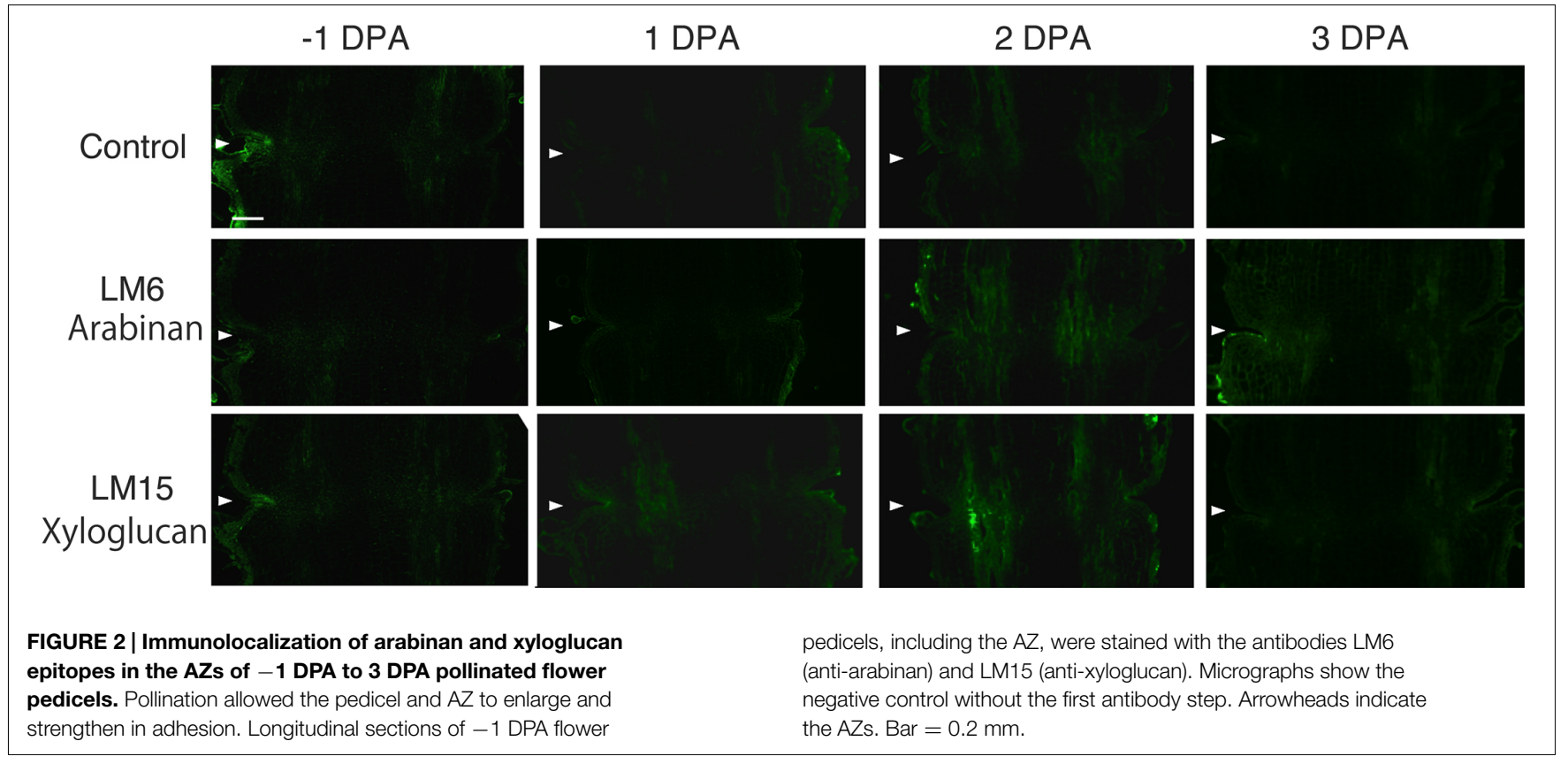

Lyloglucan
LM15
$\begin{aligned} & \text { FIGURE } 3 \text { | Immunolocalization of arabinan and xyloglucan } \\ & \text { epitopes in the AZs of }-\mathbf{1} \text { DPA to yellowing unpollinated } \\ & \text { flower pedicels. Unsuccessful pollination led to flower } \\ & \text { abscission. Longitudinal sections of }-1 \text { DPA flower pedicels, }\end{aligned}$

1 DPA, 2 DPA, 3 DPA pedicels and yellowing pedicels. XTH was determined by immunolocalization using polyclonal antibodies (Liu et al., 2013). In tomatoes, SlXTH1-25 was reported as the gene that encodes XTH (Muñoz-Bertomeu et al., 2013) and the antibody detected epitopes in all the XTH proteins examined (Takizawa et al., 2014). Expansin is a peripheral membrane protein that may cause loosening and extension of plant cell walls by disrupting non-covalent bonding between cellulose microfibrils and matrix glucans. Tomato expansins have also been reported (Rose et al., 2000), and we used commercial polyclonal antibodies that are reactive to $\beta$-expansin as shown in web page of antibody-online.com ${ }^{7}$. The epitope(s) recognized by

\footnotetext{
${ }^{7}$ http://www.antibodies-online.com/antibody/726770/anti-beta+ (Expansin+EXPB2/)
}

the anti- $\beta$-expansin is thought to occur in all tomato expansins. In the pollinated flower pedicel, the intensities of XTH and expansin epitopes were low in -1 DPA to 3 DPA (Figure 4). In the unpollinated flower pedicel, LM6 and LM15 labeling of arabinan and xyloglucan increased (Figure 3). However, during floral abscission, we observed a large 1 DPA peak in the polyclonal antibody labeling of XTH in the AZ, which then decreased (Figure 5).

Remarkably, expansin epitopes increased gradually but dramatically, specifically at the AZ, during floral abscission, whereas LM6 and LM15 labeling of arabinan and xyloglucan, respectively, also increased (Figure 3). The highest signals of expansin were detected during the yellowing stage just prior to abscission (Figure 5) 


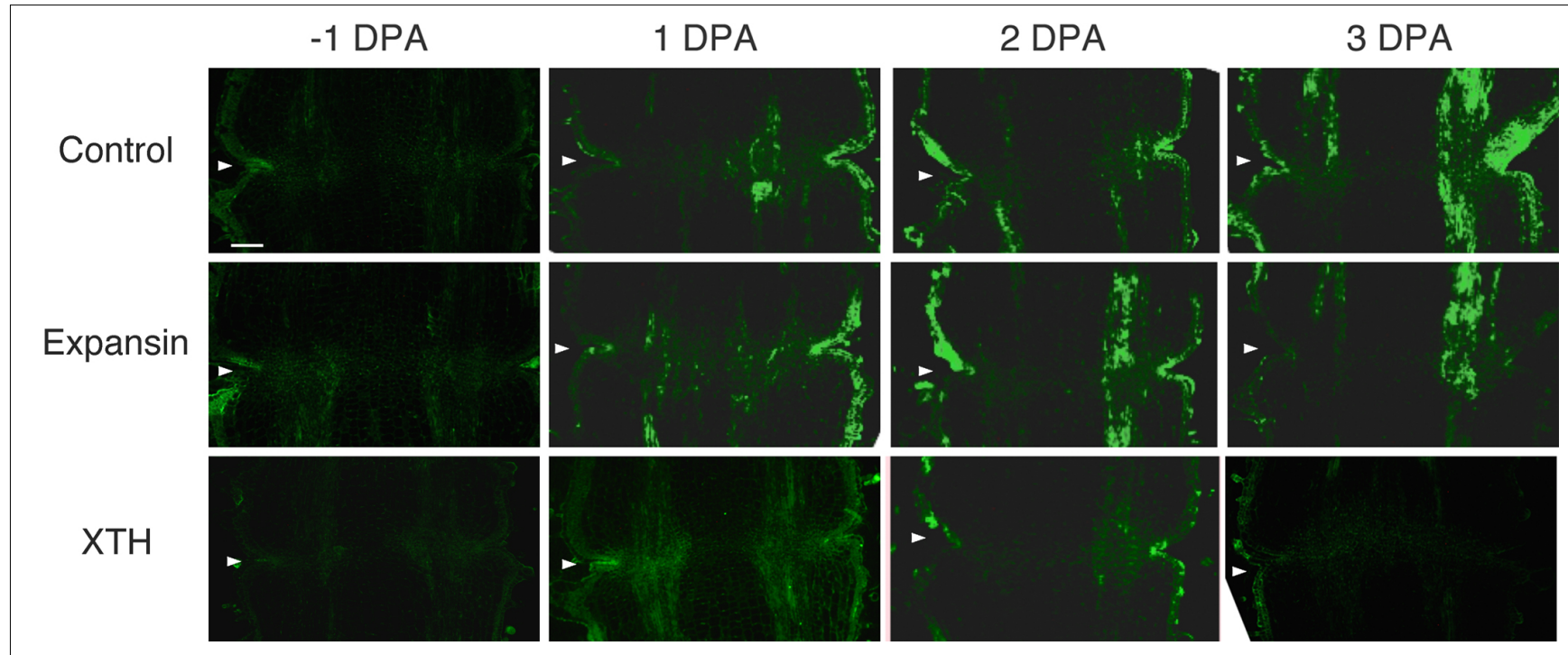

FIGURE 4 | Immunolocalization of expansin and XTH epitopes in the AZs of -1 DPA to 3 DPA pollinated flower pedicels. Pollination allowed the pedicel and AZ to enlarge and strengthen in adhesion. Longitudinal sections of
-1 DPA flower pedicels, including the AZ, were stained with the anti-expansin and anti-xyloglucan antibodies. Micrographs show the negative control without the first antibody step. Arrowheads indicate the AZs. Bar $=0.2 \mathrm{~mm}$.

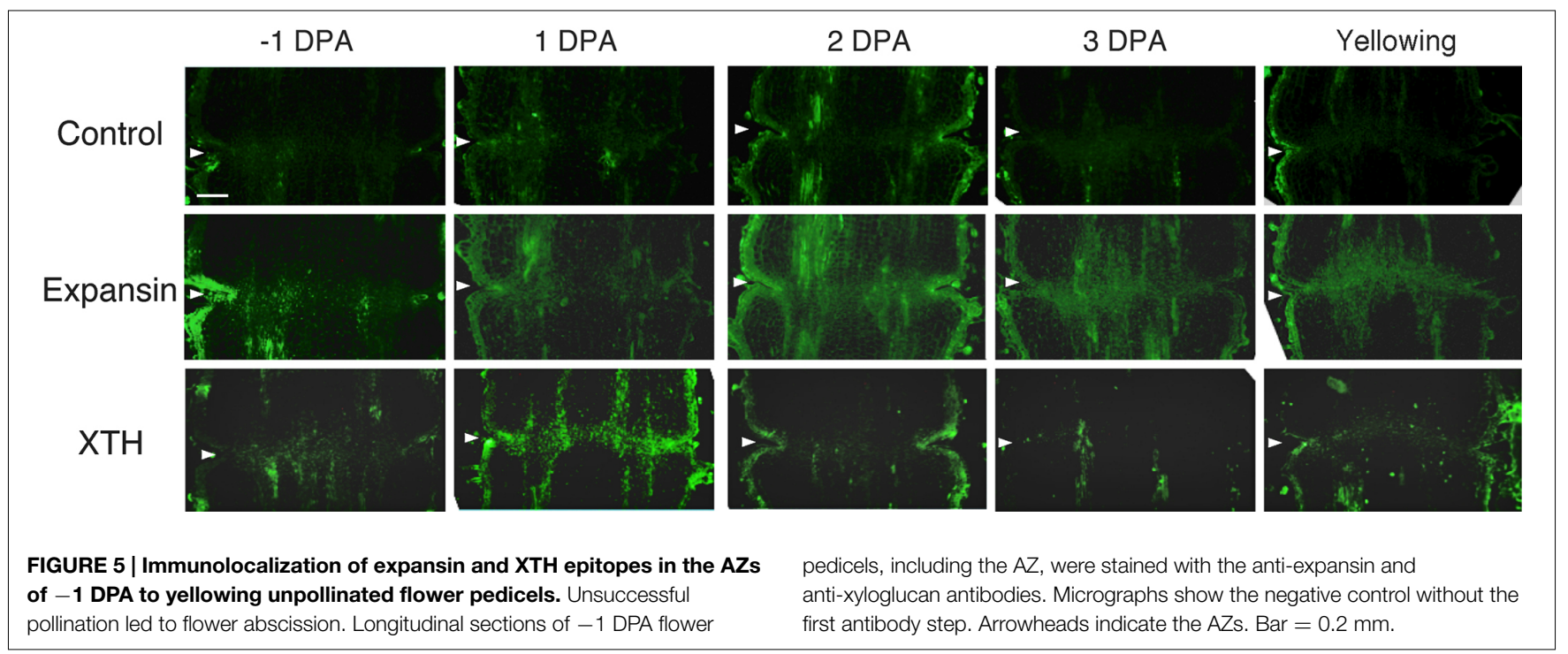

\section{Immunolocalization of XTH and Expansin Epitopes in MG Fruit Pedicels and OR Fruit Pedicels}

To determine whether flower and fruit abscission from the pedicel AZ occur by similar mechanisms, the distribution of XTH and expansin epitopes were investigated by applying the same set of polyclonal antibodies to mature green (MG) and over ripe (OR) fruit pedicel sections, each representing a stage in which adhesion at the AZ was strengthened or loosened, respectively. In MG fruit pedicels, the intensities of XTH and expansin epitope labeling were relatively low (Figure 6). We detected vascular bundles of cell wall loosening protein epitopes, but they were not as intense (Figure 6). We did not observe AZ-specific labeling of any epitopes.
The immunolabeling pattern of OR fruit pedicels did not significantly differ from that of MG fruit pedicels (Figure 6); there were high labeling intensities of both XTH and expansin in the vascular bundles (Figure 7). The dramatic increase in expansin, specifically seen at the $A Z$, of the abscising flower pedicel was not observed in the loosened AZ of the OR fruit pedicel, nor was any AZ-specific labeling detected by immunostaining.

\section{Immunolocalization of Secondary Cell wall Synthase and Lignin Staining of MG and OR Fruit Pedicels}

To determine the function of secondary CESA proteins (CESA4, CESA7, and CESA8) during fruit abscission, we used specific polyclonal antibodies to examine $\mathrm{MG}$ and $\mathrm{OR}$ fruit pedicel 


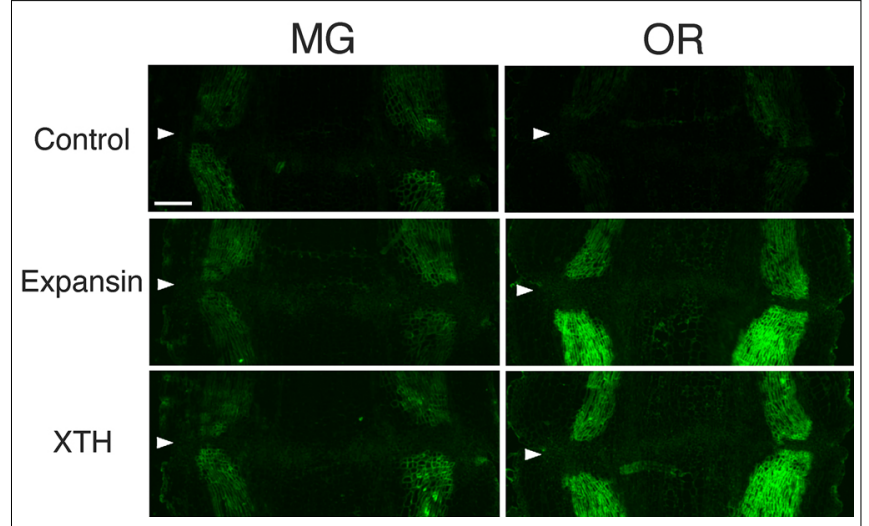

FIGURE 6 | Immunolocalization of expansin and XTH epitopes in the AZs of mature green (MG) and over-ripe (OR) fruit pedicels.

Longitudinal sections of $M G$ fruit pedicels, including the $A Z$, were stained with the anti-expansin and anti-xyloglucan antibodies. Micrographs show the negative control without the first antibody step. Arrowheads indicate the AZs. Bar $=0.5 \mathrm{~mm}$.

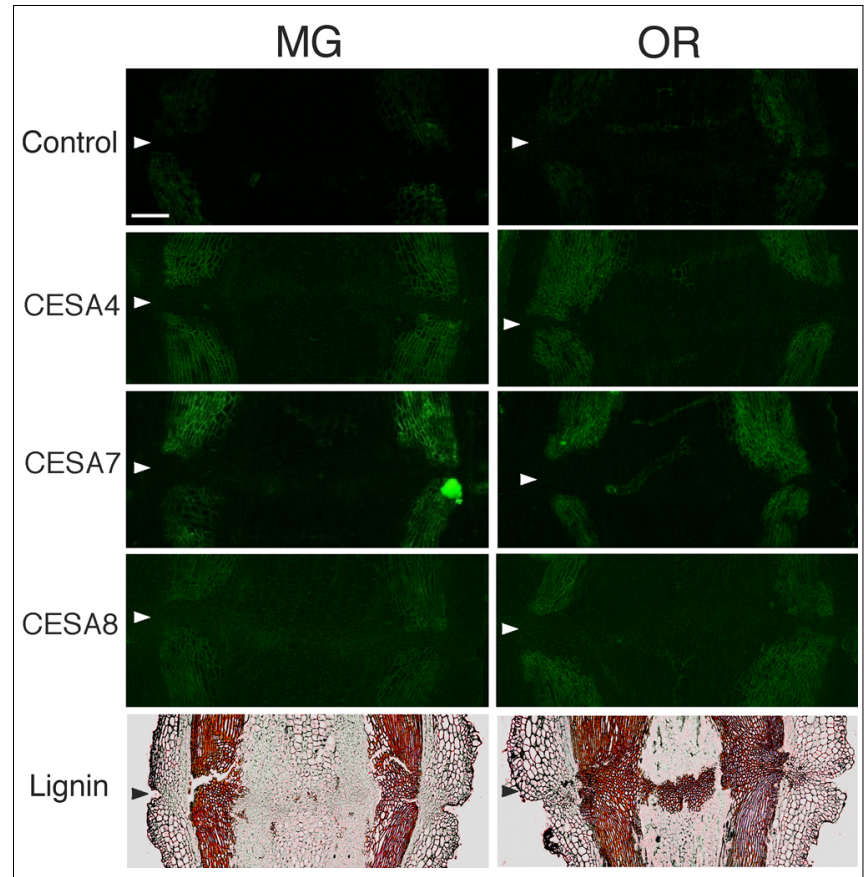

FIGURE 7 | Immunolocalization of CesA4, CesA7 and CesA8 epitopes in the AZs of mature green (MG) and over-ripe (OR) fruit pedicels.

Longitudinal sections of $M G$ fruit pedicels, including the $A Z$, were stained with the anti-CesA4, 7, and 8 antibodies. Micrographs show the negative control without the first antibody step. Phloroglucinol staining for lignin of AZs in MG and OR fruit pedicels. Arrowheads indicate the AZs. Bar $=0.5 \mathrm{~mm}$.

sections. CESA4, CESA7, and CESA8 are the most major marker enzymes for secondary cell wall synthesis (Carroll et al., 2012). The immunogens of the polyclonal antibodies of CESA4, CESA7, and CESA8 were produced from a recombinant peptide of cellulose synthase A catalytic subunit 4 (Q84JA6), 7 (Q9SWW6) and 8 (Q8LPK5), respectively ${ }^{8}$. Sequence similarity at the amino acid

\footnotetext{
${ }^{8}$ www.agrisera.com product No. AS12 2582, AS12 2581, and AS12 2580.
}

level of cellulose synthase A catalytic subunit 4, 7, and 8 are quite high compared with tomato CESA like genes (87, 91 and $88 \%$ identical, respectively), and there is one ortholog for the each CESAs in the tomato genome. Therefore, these commercial polyclonal antibodies of CESA4, 7, and 8 recognize CESA4, 7, and 8 of tomato with high probability. The results of immunostaining indicated no significant increase, decrease, or distributional change in cell wall epitopes in the AZs of loosening OR fruit pedicels. However, phloroglucinol staining was specifically observed at the AZs of the OR fruit pedicels (Figure 7). The lignin staining by phloroglucinol was not seen in the AZs of MG fruit pedicels (Figure 7), suggesting that the lignification was associated with an abscission-specific event.

\section{Discussion}

We observed natural abscission at two different stages of fruit development in the tomato pedicel: in unsuccessfully pollinated flower pedicels during the time of fruit set, and at the end of fruit development in pedicels of fully ripened fruits. However, the actual shedding event seemed to occur differently in the two scenarios (Figure 1). In OR fruit, adhesion at the AZ weakened and became vulnerable to physical stress, but was kept intact until harvested by hand, whereas in unpollinated flowers, shedding occurred naturally. We previously reported that during floral abscission, a large increase was observed in LM15 labeling of xyloglucan, specifically at the AZ in the abscised pedicel. During fruit abscission, unlike in floral abscission, no AZ-specific deposition of cell wall polysaccharides was observed. However, high autofluorescence was seen in the $\mathrm{AZ}$ of overripe fruit pedicels, suggesting secondary cell wall synthesis and lignification of the AZ prior to fruit abscission (Iwai et al., 2013). Because of the small size and limited amount of AZ samples that can be examined, enzymatic analyses of cell wall modifying activities are quite difficult, therefore we visualized the presence of XTH, expansin, and cellulose synthase using immunohistochemistry.

To analyze the changes occurring within the AZ, cell-wall polysaccharides were visualized by immunolocalization. Previous research has identified a number of cell wall-degrading enzymes, including endoglucanases (Tucker and Milligan, 1991; Tucker et al., 1991; del Campillo and Bennett, 1996; Mishra et al., 2008), polygalacturonases (Kalaitzis et al., 1995, 1997; GonzalezCarranza et al., 2002, 2007), and XTHs (Campbell and Braam, 1999) that are involved in the abscission of tomato fruits. The levels of these enzymes increase during the abscission process; therefore, we focus on the cell wall and its remodeling, which may be the target or product of enzyme activity during abscission of the reproductive organ.

At the beginning of the study, we speculated that cell wall loosening through the abscission of the xyloglucan moieties by xyloglucan endohydrolase (XEH) activity might be important. However, during floral abscission, we observed a large 1 DPA peak in the polyclonal antibody labeling of XTH in the AZ, which then decreased (Figure 5). In addition, the intensity of LM15 labeling for xyloglucan epitopes increased dramatically, preferentially at the AZ (Figure 3). These results indicate that gradual xyloglucan accumulation is important for the abscission event. XTHs were 
not detected in last stage of abscission (yellowing). This result indicates that XTH protein is degraded in the relatively short period. Similarly the rapid turnover of XTH protein was reported during the expansion of tomato fruit (Catalá et al., 2000). LeEXT1 levels were high in young expanding fruit, exhibiting a peak at the fruit expansion stage, but decreased dramatically at the expanded fruit stage. Almost all XTHs studied to date have shown endotransglucosylase activity (Vissenberg et al., 2000; Baumann et al., 2007). The increase in xyloglucan endotransglucosylase (XET) action indicates active cell wall remodellings and reconstructions of the xyloglucan related cell walls in the AZ during the $1 \mathrm{DPA}$ stage. It is likely that changes mediated by XET action may allow easier accessibility of the cell wall to other cell wall hydrolytic enzymes, thus accelerating abscission. Conversely, abscission is associated with an increase in cell size in Arabidopsis and citrus (Bleecker and Patterson, 1997; Agustí et al., 2009). XET action could be required for rearrangement of the cross-linking cell wall hemicelluloses during cell function is enlargement. This could lead to a decrease in cell wall mechanical strength, and hence abscission.

Expansin associated with the process of wall extension during cell growth ( $\mathrm{Li}$ et al., 2003). It has, however, become clear that expansins also make a significant contribution to the process of fruit softening, which involves wall breakdown, rather than expansion. It has been observed that fruit softening and abscission share a number of features in common (Rose et al., 2003). Arabidopsis expansin, AtExp10, resulted in the expression of $\beta$ glucuronidase specifically at the base of leaf petioles and silique pedicels where they join the inflorescence stem. The authors proposed that this indicated a role of AtExp10 in abscission (Cho and Cosgrove, 2000). Expansins have functions that may increase disorder in cellulose crystals, making glucan chains more susceptible to hydrolysis (Cosgrove, 1999). In our data, AZ localize expansin epitopes increased dramatically and the highest levels of expansin were detected during the yellowing stage just prior to abscission (Figure 5), These results suggest that expansins may increase disorder in cellulose crystals, making the glucan chains more susceptible to hydrolysis. This suggests that expansins play a role in abscission by promoting the degradation of the linkage between cellulose and other components in the cell wall.

A stable level of xyloglucan may be important during rapid cell expansion, which is accompanied by rapid cell wall synthesis, and suggests functions related to XTH and expansin. Cell wall remodeling through rearrangement of the wall xyloglucans by the XET action of one to several XTHs might be essential determinants in the process of abscission. And expansin increase may be a trigger function of floral abscission.

In the case of leaflet abscission in Sambucus nigra, ethylenepromoted expansin was important for undergoing cell separation

\section{References}

Agustí, J., Merelo, P., Cercós, M., Tadeo, F. R., and Talón, M. (2009). Comparative transcriptional survey between laser-microdissected cells from laminar abscission zone and petiolar cortical tissue during ethylene-promoted abscission in citrus leaves. BMC Plant Biol. 9:127. doi: 10.1186/1471-22299-127
(Belfield et al., 2005). Likewise ethylene-responsive XTH was also important for petal abscission in rose (Singh et al., 2011). Therefore, ethylene might be important for floral abscission in tomato.

The immunostaining results from both MG and OR fruit pedicel AZs were similar, showing a relatively high intensity of XTH and expansin labeling of $\mathrm{HG}$ at the epidermis in and around the vascular bundles. During fruit development and formation, the pedicel itself, as well as the vascular bundles, thickens and its diameter increases, as can be seen by comparing flower and fruit pedicel micrographs (Figures 2-5). The strong XTH and expansin labeling in the MG fruit pedicels may be due to changes in the cell walls; possibly a new cell-wall network is created by bonding xyloglucan and cellulose. The labeling intensity of cell wall modifying proteins may reflect newly created cellulose-pectin binding that occurs via xyloglucan-based side chains that may lead to the development of stronger vascular tissue for delivering large amounts of water to the developing fruit.

Although strong labeling was observed in the vascular bundles and epidermis, no AZ-specific XTH and expansin epitope labeling was observed in either the MG or OR fruit pedicel AZs (Figure 6 and 7). The dramatic deposition of expansin in the flower AZ prior to abscission was not apparent in the OR fruit AZ (Figure 6 and 7). In our previous report, no AZ-specific LM6 and LM15 epitope labeling was observed in the MG or OR fruit pedicel AZs. It showed that the intensity of arabinan and xyloglucan labeling was low in both the MG and OR pedicels (Iwai et al., 2013). However, AZ-specific lignification was observed in the AZ of the OR fruit pedicel. These results suggest that lignification was due to secondary cell wall components, including lignin and other phenolics (Figure 7). However, AZ-specific secondary CESA proteins (CESA4, CESA7, and CESA8) were not detected during fruit abscission. These results suggest AZ-specific lignification might not be associated with normal secondary cell wall synthesis. Floral abscission following unsuccessful pollination is fundamentally different from that following fruit ripening. Floral abscission occurs by a remodeling involving the deposition of cell wall polysaccharides, whereas fruit abscission occurs through lignin deposition.

The results of our study suggest that floral abscission, determined by the successfulness of pollination, and fruit abscission that occurs post-ripening, are regulated by different mechanisms-floral abscission through the remodeling of cell wall polysaccharides, and fruit shedding through lignification.

\section{Acknowledgments}

JSPS KAKENHI Grant numbers 24114006 and 25440125 financially supported this work. The Micro-Tom seeds were obtained from the National BioResource Project Tomato (NBRP-tomato).

Anjanasree, K. N., and Bansal, K. C. (2003). Isolation and characterization of ripening-related expansin cDNA from tomato. J. Plant Biochem. Biotech. 12, 31-35. doi: 10.1007/BF03263156

Baumann, M. J., Eklof, J. M., Michel, G., Kallas, A. M., Teeri, T. T., Czjzek, M., et al. (2007). III Structural evidence for the evolution of xyloglucanase activity from xyloglucan endo-transglycosylases: biological implications for cell wall metabolism. Plant Cell 19, 1947-1963. doi: 10.1105/tpc.107.051391 
Belfield, E. J., Ruperti, B., Roberts, J. A., and McQueen-Mason, S. (2005). Changes in expansin activity and gene expression during ethylene-promoted leaflet abscission in Sambucus nigra. J. Exp. Bot. 56, 817-823. doi: 10.1093/jxb/eri076

Bleecker, A. B., and Patterson, S. E. (1997). Last exit: senescence, abscission, and meristem arrest in Arabidopsis. Plant Cell 9, 1169-1179. doi: 10.1105/ tpc.9.7.1169

Brummell, D. A., Harpster, M. H., Civello, P. M., Palys, J. M., Bennett, A. B., and Dunsmuir, P. (1999). Modification of expansin protein abundance in tomato fruit alters softening and cell wall polymer metabolism during ripening. Plant Cell 11, 2203-2216. doi: 10.1105/tpc.11.11.2203

Campbell, P., and Braam, J. (1999). Xyloglucan endotransglucosylases: diversity of genes, enzymes and potential wall-modifying functions. Trends Plant Sci. 4, 361-366. doi: 10.1016/S1360-1385(99)01468-5

Carroll, A., Mansoori, N., Li, S., Lei, L., Vernhettes, S., Visser, R. G., et al. (2012). Complexes with mixed primary and secondary cellulose synthases are functional in Arabidopsis plants. Plant Physiol. 160, 726-737. doi: 10.1104/pp.112. 199208

Catalá, C., Rose, J. C. and Bennett, A. B. (1997). Auxin regulation and spatial localization of an endo-1, 4- $\beta$-D-glucanase and a xyloglucan endotransglucosylase in expanding tomato hypocotyls. Plant J. 12, 417-426. doi: 10.1046/j.1365313X.1997.12020417.x

Catalá, C., Rose, J. K., and Bennett, A. B. (2000). Auxin-regulated genes encoding cell wall-modifying proteins are expressed during early tomato fruit growth. Plant Physiol. 122, 527-534. doi: 10.1104/pp.122.2.527

Catalá, C., Rose, J. C., York, W. S., Albersheim, P., Darvill, A. G. and Bennett, A. B. (2001). Characterization of a tomato xyloglucan endotransglucosylase gene that is down-regulated by auxin in etiolated hypocotyls. Plant Physiol. 127, 1180-1192. doi: 10.1104/pp.010481

Chen, F., and Bradford, K. J. (2000). Expression of an expansin is associated with endosperm weakening during tomato seed germination. Plant Physiol. 124, 1265-1274. doi: 10.1104/pp.124.3.1265

Cho, H. T., and Cosgrove, D. J. (2000). Altered expression of expansin modulates leaf growth and pedicel abscission in Arabidopsis thaliana. Proc. Natl. Acad. Sci. U.S.A. 97, 9783-9788. doi: 10.1073/pnas.160276997

Cosgrove, D. J. (1999). Enzymes and other agents that enhance cell wall extensibility. Ann. Rev. Plant Physiol. Plant Mol. Biol. 50, 391-417. doi: 10.1146/annurev.arplant.50.1.391

Cosgrove, D. J., Li, L. C., Cho, H. T., Hoffmann-Benning, S., Moore, R. C., and Blecker, D. (2002). The growing world of expansins. Plant Cell Physiol. 43, 1436-1444. doi: 10.1093/pcp/pcf180

del Campillo, E., and Bennett, A. (1996). Pedicel break strength and cellulase gene expression during tomato flower abscission. Plant Physiol. 111, 813-820. doi: 10.1104/pp.111.3.813

de Silva, J., Jarman, C. D., Arrowsmith, D. A., Stronach, M. S., Chengappa, S., Sidebottom, C., et al. (1993). Molecular characterization of a xyloglucan specific endo- $(1,4)-\beta$-D-glucanase (xyloglucan endotransglucosylase) from nasturtium seeds. Plant J. 3, 701-711. doi: 10.1111/j.1365-313X.1993.00701.x

Fry, S. C. (1989). The structure and functions of xyloglucan. J. Exp. Bot. 40, 1-11. doi: $10.1093 / \mathrm{jxb} / 40.1 .1$

Fry, S. C., Smith, R. C., Renwick, K. F., Martin, D. J., Hodge, S. K., and Matthews, K. J. (1992). Xyloglucan endotransglycosylase, a new wall loosening enzyme activity from plants. Biochem. J. 282, 821-828.

Gonzalez-Carranza, Z. H., Whitelaw, C. A., Swarup, R., and Roberts, J. A. (2002). Temporal and spatial expression of a polygalacturonase during leaf and flower abscission in oilseed rape and Arabidopsis. Plant Physiol. 128, 534-543. doi: 10.1104/pp.010610

Gonzalez-Carranza, Z. H., Elliott, K. A. and Roberts, J. A. (2007). Expression of polygalacturonases and evidence to support their role during cell separation processes in Arabidopsis thaliana. J. Exp. Bot. 58, 3719-3730. doi: $10.1093 /$ jxb/erm 222

Hayashi, T. (1989). Xyloglucans in the primary cell wall. Ann. Rev. Plant Physiol. Plant Mol. Biol. 40, 139-168. doi: 10.1146/annurev.pp.40.060189.001035

Ishimaru, M., and Kobayashi, S. (2002). Expression of a xyloglucan endotransglycosylase gene is closely related to grape berry softening. Plant Sci. 162, 621-628. doi: 10.1016/S0168-9452(01)00608-2

Iwai, H., Terao, A., and Satoh, S. (2013). Changes in distribution of cell wall polysaccharides in floral and fruit abscission zones during fruit development in tomato (Solanum lycopersicum). J. Plant Res. 126, 427-437. doi: 10.1007/s10265012-0536-0
Kalaitzis, P., Koehler, S. M., and Tucker, M. L. (1995). Cloning of a tomato polygalacturonase expressed in abscission. Plant Mol. Biol. 28, 647-656. doi: $10.1007 / B F 00021190$

Kalaitzis, P., Solomos, T., and Tucker, M. L. (1997). Three different polygalacturonases are expressed in tomato leaf and flower abscission, each with a different temporal expression pattern. Plant Physiol. 113, 1303-1308. doi: 10.1104/pp.113.4.1303

Lee, Y., Choi, D., and Kende, H. (2001). Expansins: ever-expanding numbers and functions. Curr. Opin. Plant Biol. 4, 527-532. doi: 10.1016/S13695266(00)00211-9

Li, Y., Jones, L., and McQueen-Mason, S. J. (2003). Expansins and plant cell growth. Curr. Opin. Plant Biol. 6, 603-610. doi: 10.1016/j.pbi.2003.09. 003

Liu, L., Shang-Guan, K., Zhang, B., Liu, X., Yan, M., Zhang, L., et al. (2013). Brittle Culm1, a COBRA-Like protein, functions in cellulose assembly through binding cellulose microfibrils. PLoS Genet. 9:e1003704. doi: 10.1371/journal.pgen.1003704

Mao, L., Begum, D., Chuang, H., Budiman, M. A., Szymkowiak, E. J., Irish, E. E., et al. (2000). JOINTLESS is a MADS-box gene controlling tomato flower abscission zone development. Nature 406, 910-913. doi: 10.1038/35022611

Marcus, S. E., Verhertbruggen, Y., Hervé, C., Ordaz-Ortiz, J. J., Farkas, V., Pedersen, H. L., et al. (2008). Pectic homogalacturonan masks abundant sets of xyloglucan epitopes in plant cell walls. BMC Plant Biol. 8:60. doi: 10.1186/1471-22298-60

Mella, R. A., Burgin, M. J., and Sanchez, R. A. (2004). Expansin gene expression in Datura ferox L. seeds is regulated by the low-fluence response, but not by the high-irradiance response, of phytochromes. Seed Sci. Res. 14, 61-71. doi: 10.1079/SSR2003155

McQueen-Mason, S. J., and Cosgrove, D. J. (1994). Disruption of hydrogenbonding between plant-cell wall polymers by proteins that induce wall extension. Proc. Natl. Acad. Sci. U.S.A. 91, 6574-6578. doi: 10.1073/pnas.91.14. 6574

McQueen-Mason, S. J., and Cosgrove, D. J. (1995). Expansin mode of action on cell walls: analysis of wall hydrolysis, stress-relaxation, and binding. Plant Physiol. $107,87-100$.

McQueen-Mason, S. J., Durachko, D. M., and Cosgrove, D. J. (1992). Two endogenous proteins that induce cell-wall extension in plants. Plant Cell 4, 1425-1433. doi: $10.1105 /$ tpc.4.11.1425

Mishra, A., Khare, S., Trivedi, O. K., and Nath, P. (2008). Ethylene induced cotton leaf abscission is associated with higher expression of cellulase (GhCel1) and increased activities of ethylene biosynthesis enzymes in abscission zone. Plant Physiol. Biochem. 46, 54-63. doi: 10.1016/j.plaphy.2007.09.002

Muñoz-Bertomeu, J., Miedes, E. and Lorences, E. P. (2013). Expression of xyloglucan endotransglucosylase/hydrolase (XTH) genes and XET activity in ethylene treated apple and tomato fruits. J. Plant Physiol. 170, 1194-1201. doi: 10.1016/j.jplph.2013.03.015

Nishikubo, N., Awano, T., Banasiak, A., Bourquin, V., Ibatullin, F., Funada, R., et al. (2007). Xyloglucan endo-transglycosylase (XET) functions in gelatinous layers of tension wood fibers in poplar: a glimpse into the mechanism of the balancing act of trees. Plant Cell Physiol. 48, 843-855. doi: 10.1093/pcp/pcm055

Nishikubo, N., Takahashi, J., Roos, A. A., Derba-Maceluch, M., Piens, K., Brumer, H., et al. (2011). Xyloglucan endo-transglycosylase-mediated xyloglucan rearrangements in developing wood of hybrid aspen. Plant Physiol. 155, 399-413. doi: $10.1104 /$ pp.110.166934

Nishitani, K., and Tominaga, R. (1992). Endo-xyloglucan transferase, a novel class of glycosyltransferase that catalyzes transfer of a segment of xyloglucan molecule to another xyloglucan molecule. J. Biol. Chem. 267, 21058-21064.

Orfila, C., and Knox, J. P. (2000). Spatial regulation of pectic polysaccharides in relation to pit fields in cell walls of tomato fruit pericarp. Plant Physiol. 122, 775-781. doi: 10.1104/pp.122.3.775

Potter, I., and Fry, S. C. (1994). Changes in xyloglucan endotransglucosylase (XET) activity during hormone-induced growth in lettuce and cucumber hypocotyls and spinach cell suspension cultures. J. Exp. Bot. 45, 1703-1710.

Roberts, J. A., Elliott, K., and Gonzalez-Carranza, Z. H. (2002). Abscission, dehiscence, and other cell separation processes. Ann. Rev. Plant Biol. 53, 131-158. doi: 10.1146/annurev.arplant.53.092701.180236

Roberts, J. A., Whitelaw, C. A., Gonzalez-Carranza, Z. H., and McManus, M. T. (2000). Cell separation processes in plants-models, mechanisms and manipulation. Ann. Bot. 86, 223-235. doi: 10.1006/anbo.2000.1203 
Rose, J. C., and Bennett, A. B. (1999). Cooperative disassembly of the cellulose-xyloglucan network of plant cell walls: parallels between cell expansion and fruit ripening. Trends Plant Sci. 4, 176-183. doi: 10.1016/S1360-1385(99) 01405-3

Rose, J. K., Cosgrove, D. J., Albersheim, P., Darvill, A. G. and Bennett, A. B. (2000). Detection of expansin proteins and activity during tomato fruit ontogeny. Plant Physiol. 123, 1583-1592. doi: 10.1104/pp.123.4.1583

Rose, J. C., Braam, J., Fry, S. C., and Nishitani, K. (2002). The XTH family of enzymes involved in xyloglucan endotransglucosylation and endohydrolysis: current perspectives and a new unifying nomenclature. Plant Cell Physiol. 43, 1421-1435. doi: 10.1093/pcp/pcf171

Rose, J. C., Catala, C., Gonzalez-Carranza, Z. H., and Roberts, J. A. (2003). "Cell wall disassembly," in The Plant Cell Wall, ed. J. K. C. Rose (Oxford: Blackwell Publishing), 264-324.

Rose, J. C., Lee, H. H., and Bennett, A. B. (1997). Expression of a divergent expansin gene is fruit-specific and ripening-regulated. Proc. Natl. Acad. Sci. U.S.A. 94, 5955-5960. doi: 10.1073/pnas.94.11.5955

Saab, I., and Sachs, M. (1996). A flooding-induced xyloglucan endotransglucosylase homologue in maize is responsive to ethylene and associated with aerenchyma. Plant Physiol. 112, 385-391. doi: 10.1104/pp.112.1.385

Saladié, M., Rose, J. C., Cosgrove, D. J., and Catala, C. (2006). Characterization of a new xyloglucan endotransglucosylase/hydrolase $(\mathrm{XTH})$ from ripening tomato fruit and implications for the diverse modes of enzymic action. Plant J. 47, 282-295. doi: 10.1111/j.1365-313X.2006.02784.x

Schroder, R., Atkinson, R. G., Langenkamper, G., and Redgwell, R. J. (1998). Biochemical and molecular characterization of xyloglucan endotransglucosylase from ripe kiwifruit. Planta 204, 242-251. doi: 10.1007/s004250050253

Schunmann, P. H. D., Smith, R. C., Lang, V., Matthews, P. R., and Chandler, P. M. (1997). Expression of XET-related genes and its relation to elongation in leaves of barley (Hordeum vulgare L.). Plant Cell Environ. 20, 1439-1450. doi: 10.1046/j.1365-3040.1997.d01-49.x

Singh, A. P., Tripathi, S. K., Nath, P., and Sane, A. P. (2011). Petal abscission in rose is associated with the differential expression of two ethylene-responsive xyloglucan endotransglucosylase/hydrolase genes, RbXTH1 and RbXTH2.J. Exp. Bot. 62, 5091-5103. doi: 10.1093/jxb/err209

Smith, R. C., and Fry, S. C. (1991). Endotransglycosylation of xyloglucans in plant cell suspension cultures. Biochem. J. 279, 529-535.

Tadeo, F. R. and Primo-Millo, E. (1990). Peroxidase activity changes and lignin deposition during the senescence process in Citrus stigmas and styles. Plant Sci. 68, 47-56. doi: 10.1016/0168-9452(90)90151-D

Takizawa, A., Hyodo, H., Wada, K., Ishii, T., Satoh, S., and Iwai, H. (2014). Regulatory specialization of xyloglucan and glucuronoarabinoxylan in pericarp cell walls during fruit ripening in tomato (Solanum lycopersicum). PLoS ONE 9:e89871. doi: 10.1371/journal.pone.0089871

Taylor, J. E., Tucker, G. A., Lasslett, Y., Smith, C. J. S., Arnold, C. M., Watson, C. F., et al. (1991). Polygalacturonase expression during leaf abscission of normal and transgenic tomato plants. Planta 183, 133-138. doi: 10.1007/BF00197577

Tucker, M. L., and Milligan, S. B. (1991). Sequence analysis and comparison of avocado fruit and bean abscission cellulases. Plant Physiol. 199, 928-933. doi: 10.1104/pp.95.3.928

Tucker, M. L., Baird, S. L. and Sexton, R. (1991). Bean leaf abscission: tissue specific accumulation of a cellulase mRNA. Planta 186, 52-57. doi: 10.1007/BF00201497

Vissenberg, K., Fry, S. C., and Verbelen, J.-P. (2001). Root hair initiation is coupled to highly localized increase of xyloglucan endotransglycosylase action in Arabidopsis roots. Plant Physiol. 127, 1125-1135. doi: 10.1104/pp.010295

Vissenberg, K., Martinez-Vilchez, I. M., Verbelen, J. P., Miller, J. G., and Fry, S. C. (2000). In vivo colocalization of xyloglucan endotransglycosylase activity and its donor substrate in the elongation zone of Arabidopsis roots. Plant Cell 12, 1229-1237. doi: 10.1105/tpc.12.7.1229

Whitney, S. E. C., Gidley, M. J., and McQueen-Mason, S. J. (2000). Probing expansin action using cellulose/hemicellulose composites. Plant J. 22, 327-334. doi: 10.1046/j.1365-313x.2000.00742.x

Willats, W. G. T., Marcus, S. E., and Knox, J. P. (1998). Generation of a monoclonal antibody specific to (1->5)-a-L- arabinan. Carbohydr. Res. 308, 149-152. doi: 10.1016/S0008-6215(98)00070-6

Xu, W., Campbell, P., Vargheese, A. K., and Braam, J. (1996). The Arabidopsis XET related gene family: environmental and hormonal regulation of expression. Plant J. 9, 879-889. doi: 10.1046/j.1365-313X.1996.9060879.x

Yokoyama, R., Rose, J. K., and Nishitani, K. (2004). A surprising diversity and abundance of xyloglucan endotransglucosylase/hydrolases in rice. Classification and expression analysis. Plant Physiol. 134, 1088-1099. doi: 10.1104/pp.103. 035261

Conflict of Interest Statement: The authors declare that the research was conducted in the absence of any commercial or financial relationships that could be construed as a potential conflict of interest.

Copyright (c) 2015 Tsuchiya, Satoh and Iwai. This is an open-access article distributed under the terms of the Creative Commons Attribution License (CC BY). The use, distribution or reproduction in other forums is permitted, provided the original author(s) or licensor are credited and that the original publication in this journal is cited, in accordance with accepted academic practice. No use, distribution or reproduction is permitted which does not comply with these terms. 